\title{
altrelettere
}

\author{
Natalia Costa-Zalessow
}

\section{Alla scoperta di Margherita Costa}

\begin{abstract}
In questo saggio, letto per l'apertura del Convegno Internazionale di Studi (L'Aquila, 12 aprile 2021), traccio la storia della mia scoperta della poetessa barocca Margherita Costa, che portò alla pubblicazione dell'antologia da me curata, Voice of a Virtuosa and Courtesan, Selected Poems of Margherita Costa, in cui offro una scelta delle sue poesie più notevoli. Margherita rimase dimenticata per molto tempo e non fu capita dai pochi critici che si occuparono di lei, incluso Benedetto Croce. Ma dopo aver letto i suoi quattordici libri, pubblicati fra il 1638 e il 1654, scoprii una scrittrice non solo prolifica ma versatile e originale, che coltivò diversi generi letterari, inclusi l'umorismo, l'ironia e la satira, e che osò gareggiare e polemizzare con i marinisti. Margherita Costa è l'unica poetessa italiana veramente barocca. Per illustrare queste mie conclusioni, do una serie di esempi di temi originali utilizzati da Margherita.
\end{abstract}

The e-journal «altrelettere» is hosted at the URL: http://www.altrelettere.uzh.ch, in accordance with the Open Access Policy of the University of Zurich. Please cite this article as follows: N. CosTA-ZALESSOW, Alla scoperta di Margherita Costa, in «altrelettere», (2021), pp. 13-26, DOI: 10.5903/al_uzh-51.

(C) This article is licensed under a Creative Commons Attribution 2.5. Switzerland (CC BY-NC-ND 2.5). Please read the license terms on the website: http://creativecommons.org/licenses/by-nc-nd/2.5/ch/deed.en 
Il mio primo incontro con la poetessa romana del Seicento, Margherita Costa, risale al lontano 1980, quando raccoglievo il materiale per l'antologia Scrittrici italiane dal XIII al XX secolo, uscita poi nel gennaio del 1982. Mi ero accorta che c'erano tante belle poesie nella prima raccolta di rime di Margherita, $L a$ chitarra (1638), nonostante la critica severa di Benedetto Croce che aveva liquidato la nostra poetessa con una frase sola: «Delle cantanti, e di non buona fama, fu la romana Margherita Costa, che scombiccherò ogni sorta di opere, liriche, drammi, poemi, prose, e risplende soprattutto per la sua incultura, da lei stessa dichiarata in versacci di questa sorta» (Croce 2003, 171-72), citando poi le terzine umoristiche in cui Margherita dice male dei propri versi e aggiungendo in nota che le stesse si possono leggere nella raccolta della Bergalli. Vuol dire che Croce aveva letto solo quel poco raccolto in quell'antologia? Io direi di sì, perché se avesse almeno letto le poesie del volume La chitarra sarebbe arrivato ad una conclusione diversa. Inoltre, non aveva capito che i versi da lui citati come prova che Margherita era conscia della sua ignoranza, erano invece una versione barocca e umoristica della tendenza di tutte le poetesse italiane che precedono Margherita (e di quelle che la seguiranno), a dichiarare che il loro stile era umile, rozzo o imperfetto a paragone di quello dei poeti maschi. Era una strategia per non essere accusate di volersi mettere sullo stesso piedistallo ed evitare di provocare gli uomini che preferivano la donna sottomessa anche nelle lettere.

Una volta uscita l'antologia avevo pensato di approfondire le ricerche su Margherita, ma, distratta da altri lavori, ci ritornai solo al principio del ventunesimo secolo, per merito del collega Albert N. Mancini che lavorava sui poeti e drammaturghi italiani del Seicento, per una collana americana di dizionari letterari. Mancini mi chiese di suggerirgli quali scrittrici includere. Fra quelle che gli proposi c'erano Francesca Turini Bufalini e Margherita Costa, allora poco conosciute. Fui allora invitata a scrivere le voci relative a queste autrici per il Dictionary of Literary Biography, Vol. 339: Seventeenth-Century Italian Poets and Dramatists, a cura di Albert N. Mancini e Glenn Palen Pierce, che uscì nel 2008. Questi contributi risvegliarono in me il desiderio di approfondire le ricerche e di far conoscere il valore letterario di queste due poetesse. E così videro la luce i due libri, Autobiographical Poems di Francesca Turini Bufalini, edizione bilingue, con traduzioni di Joan E. Borrelli, uscito nel 
2009, e Voice of a Virtuosa and Courtesan. Selected Poems of Margherita Costa, apparso nel 2015, anche questo bilingue con traduzioni di Joan E. Borrelli.

Dei quattordici libri pubblicati da Margherita Costa solo la commedia $\mathrm{Li}$ buffoni ha avuto un'edizione moderna, inclusa nel secondo volume di Commedie dell'Arte, curate da Siro Ferrone per Mursia, nel 1985-1986, commedia poi decifrata da Teresa Megale nell'articolo La commedia decifrata: metamorfosi e rispecchiamenti in Li buffoni di Margherita Costa, saggio essenziale per capire questa pièce, detta ridicola dalla drammaturga stessa. ${ }^{1}$

Trovandomi davanti all'assurda critica di Croce citata sopra, mi misi a leggere tutte le opere pubblicate da Margherita, dalla prima all'ultima pagina, e scoprii una scrittrice versatile, originale, veramente barocca, che ebbe il coraggio di trattare temi nuovi legati alla vita difficile delle donne e di gareggiare, o far polemica con i suoi contemporanei, incluso Marino. Inoltre, era la prima poetessa italiana ad usare l'umorismo, l'ironia, nonché la satira nelle opere da lei pubblicate.

Nel mio libro, Voice of a Virtuosa and Courtesan, offro una scelta dei versi di Margherita, che ho tratto da vari volumi, dando la preferenza alle poesie, o alle parti di poesie, che mi sembravano più notevoli, seguendo un ordine cronologico, secondo le date di pubblicazione delle raccolte, e mantenendo l'ordine interno di ogni libro. In questa sede vorrei discutere alcune poesie secondo il tema che le lega, iniziando da quelle che riguardano la vita muliebre.

Fra i Capitoli de La chitarra (1638) spicca quello intitolato Donna all'amante Tirsi, lontana da lui e gravida, in cui la donna, rimasta incinta, si lamenta della sua sorte e di quella del bambino che nascerà, condannato alla vita difficile del bastardo:

$[\ldots]$

dell'innocente piango il crudo affanno, le mie miserie e di te padre il danno.

Pargoletto lo bramo e poi m'annoia

d'averlo a partorir su queste arene qual nuovo Ascanio all'incendio di Troia disredato da te d'ogni suo bene. 
Bramo che nato appena in sen mi moia per non mirarlo involto in maggior pene, e se talvolta in sen lo sento, oh dio, figlio lo chiamo d'ogni danno mio.

Ahi, qual sventura e qual acerbo fato mi rende madre a sventurato figlio? Qual piacque al Cielo di cangiarmi stato? Qual sorte mi condusse a te al periglio? O povero innocente, a che sei nato? Chi fia ch' al viver tuo porga consiglio? La madre t'abbandona e t'è rubella. $\mathrm{Al}$ padre t'allontana iniqua stella.

Infelice pupillo, e dove sono quelle grandezze che goder dovei? Dunque alla tua 'nnocenza alcun perdono ria Fortuna non ha, non hanno i dei? $(V V C \text {, pp. } 59 \text { e } 60)^{2}$

Quante donne, attraverso i secoli, sono state sedotte e abbandonate, appena incinte, da uomini che non si curano dei figli nati e della loro sorte? Eppure Margherita è la prima poetessa a trattare questo problema come tema poetico e proponendo un'accusa indiretta. Similmente in Tirsi trafitto (del volume $L a$ selva di cipressi, 1644), descrive la penosa sorte degli orfani, osservando giustamente che la situazione è anche più grave per le bambine che per i maschi:

E tu, misera, più dell'innocente pupillo, in cui la sorte è più proterva, te, delusa bambina, egra e dolente, a peggior sorte il tuo destin conserva. [...]

Ah, quanto meglio per tuo fato imbelle era non nascer tra cotanti affanni, se misera sorgesti a crude stelle, soggetta nel più bel dei tuoi prim' anni. Non nata, il ciel ti minacciò procelle e nell'alvo ti scorse ai primi danni. Ed ora, appena fuor del sen materno resti tra fasce ancor senza governo. (VVC, pp. 185 e 187)

In questi due componimenti il tono è triste, tragico, ma più spesso Margherita esprime la sua critica in tono scherzoso, ironico, rivolgendosi alle donne, come 
per esempio in Bella donna di scherzo alle donne (del volume La chitarra), dove consiglia alle donne di non amare un uomo solo, soprattutto se geloso, che le tiene chiuse in casa. Lo scherzo è una critica delle leggi imposte alle donne ma non agli uomini:

Il dar legge alle donne è scioccheria,

Ognun faccia a suo modo, ch' in amore poco giovan le leggi senza il core.

(VVC, p. 63)

Similmente consiglia alle donne di essere generose con i baci per non morire accoltellate, come è successo a una poveraccia (fattarello di cronaca nera), la quale aveva schernito un corteggiatore. Ovviamente Margherita prende di mira la violenza maschile contro le donne, le quali pagano sempre i danni se osano opporsi alla volontà di un uomo:

Provi, negando un bacio, il tuo morire e d'un bacio negato paghi il fio.

(VVC, p. 65)

La poetessa prende in giro anche la tradizionale norma che le donne debbano occuparsi solo della casa, spesso sintetizzata nel concetto del filare, da lei usato ripetutamente:

No, no, donne, non più, non più penare, l'arte del far l'amor vada in oblio, ché l'arte nostra è solo di filare. (VVC, p. 85)

Il tema è elaborato con maggior brio nella conclusione in un'altra poesia:

Donne, al fin mi riduco

di lasciar ogni stile, ogn'altro affare,

e sol darmi al filare, poiché conosco al fine che l'altre imprese son nostre rovine.

Donna nacqui e di donna 
vestir mi voglio ogn'uso,

e sol sarà mio spasso

spassarmi ora con l'ago, or con il fuso.

Sì, sì, tutte volgete

il pensiero e il desire

al filare e al cucire.

E giacché di filar solo ci tocca,

lasciamo ogn'altro affar, prendiam la rocca.

(VVC, p. 87)

Nella canzonetta, Donne mie, poi ch' ho provato, il filare, ripetuto nell'ultimo verso delle prime quattro strofe, fa parte anche di un intreccio stilistico di parole: ma sol godo di filare - e sol bramo di filare - il cucire col filare - il cucire col filare, per concludere nella quinta strofa con:

sì, sì, donne, se volete,

di filar solo godete,

ché la donna sol dèe fare

l'esercizio del filare.

(VVC, pp. 89 e 91)

Lontano da ogni tono scherzoso sono invece i versi in cui Margherita espone le sofferenze di una cantante, versi in cui si riflette una tristezza profonda (forse autobiografica), espressa con stile notevole, molto vicino a quello usato dai poeti del periodo romantico:

Quante volte sciogliei la voce al canto

che di pianger bramai le mie sventure, e coi sospir miei rattenni il pianto, sclamando le passate mie venture.

Ahi, quante volte il mio passato vanto mi ramentai fra scene così dure, e quante e quante volte in volto finsi d'esser contenta e la mia pena vinsi.

Ridea la bocca e mi piangeva il core.

Parlaa la lingua, il mio desir tacea.

Nel mio riso copriva il mio dolore, e le miserie mie, saggia, ascondea.

(VVC, p. 37) 
A questa categoria di poesie muliebri, o protofemministe, si dovrebbero aggiungere i molti riferimenti autobiografici, i lamenti di Margherita per la sua cattiva sorte: nata povera, carriera difficile, e nessun riconoscimento letterario. Ma l'autobiografismo è anche una categoria a sé. C'è il lungo componimento in ottava rima, Elisa infelice (del volume La selva di cipressi, 1640), in cui Margherita descrive parte della sua vita, ma in modo vago e astratto, e che include una visita alla corte di Apollo, dove Elisa incontra anche i poeti e le poetesse italiani:

Per la corte s'aggira il vecchio Dante, d'anni e d'opre gravoso; avvi il Petrarca, il Bembo, il Guidaccioni e 'l Casa inante, che fan corona al lucido monarca. V'è la Sarrocchi e in nobile sembiante, la Colonnese, e a scorno della Parca la Gambara s'avanza, e 'l sesso frale3 nei suoi rai di virtù splende immortale (VVC, pp. 199 e 201)

Molte lamentele di carattere autobiografico sono sparse un po' dappertutto, anche nelle dediche a personaggi illustri delle sue opere, o nelle poesie rivolte ad amici, e alludono alla sua difficile vita, incluso l'esilio da Roma (tema, questo, che meriterebbe un'indagine a parte, che non si può fare qui).

Passiamo perciò al coraggio di Margherita nel voler gareggiare e polemizzare con i poeti barocchi, a cominciare da Marino. Nella sua seconda raccolta di versi, Il violino (1638), spicca l'idillio Violentamento di Lilla, in cui il critico Martino Capucci vide solo «un'aperta lubricità che si direbbe professionale» (CAPUCCI 1984, 233) senza accorgersi che si tratta di un'imitazione tematica del componimento erotico Trastulli estivi (in La lira) di Marino, ma con una conclusione ben diversa. Come indica già il titolo, per Marino approfittarsi di una donna addormentata è un divertimento, una conquista che soddisfa l'uomo. Margherita invece lo chiama giustamente violentamento, perché l'uomo usa la forza per possedere la donna. Contrariamente a Marino, che sostiene che la donna fosse consenziente perché aveva protestato poco (opinione ancora in voga fra molti), Margherita toglie ogni colpa a Lilla e la lascia libera di scegliersi un amante di suo gusto, 
rifiutando l'offerta di matrimonio del violentatore. Si tratta di un concetto moderno, se pensiamo che in Italia la legge che abolisce il matrimonio riparatore è datata 5 agosto 1981 .

Leggendo, nel poema Cecilia martire (1644), le ottave in cui Margherita descrive il caos creato dalle forze diaboliche nel bagno della santa per farla morire, ci troviamo davanti a infinite variazioni del movimento delle fiamme e delle acque bollenti, che ci ricordano la tecnica stilistica usata da Marino nelle ottave dedicate al canto dell'usignolo in Adone (Canto VII, vv. 32-37): stile che lei imita con elementi diversi, più eccessivi, creando versi che sono un esempio di bravura barocca.

Un'altra gara letteraria è quella con Alessandro Adimari che, nel 1637 aveva pubblicato La Tersicore, o Scherzi e paradossi poetici sopra la beltà delle donne, un volume di 50 sonetti ciascuno dedicato a una donna con un difetto diverso: troppo magra, troppo grassa, troppo alta, troppo bassa, sorda, muta, gobba, ecc., ma amabile lo stesso. Margherita, in risposta, compose Amante di donna brutta loda le sue bruttezze, l'idillio (incluso in Il Violino) in cui attribuisce, in modo burlesco, tutti questi difetti ed altri a una sola donna, vecchia e di aspetto sgradevole ma amata lo stesso dal suo amante, e supera tutti i poeti realistici che sottolineavano la bruttezza delle donne per sfogare la loro misoginia. Il linguaggio usato da Margherita è grossolano ma non offende perché è tanto eccessivo da far solo ridere o meravigliare.

Un ottimo esempio del genere satirico coltivato da Margherita è poi il Cortegiano ravveduto, satira pungente della vita di corte, dove regnano gli intrighi, le bugie, le maldicenze, la perfidia e la slealtà dei cortigiani in lotta per l'attenzione del principe. La severità della critica e il linguaggio forte della poetessa ci fanno domandare come avesse trovato il coraggio di includere questi versi in Lo stipo (1639), un libro dedicato al Principe Lorenzo de' Medici.4

Essendo un componimento di 62 ottave, non mi fu possibile includerlo nella mia antologia, ma ritenendolo un testo notevole ne preparai un'edizione commentata, che spedii ad Antonio Lanza, anche se con poca speranza di vederlo accettato, sia per la lunghezza, sia per essere un'opera di scrittrice poco conosciuta. Ma al Professor Lanza piacque e lo pubblicò in Letteratura Italiana Antica. 
All'accusa che scrivesse spesso su temi comunemente diffusi nel suo periodo si può ribadire che, sebbene a volte ciò sia vero, Margherita lo faceva con originalità, e forse meglio di tanti suoi contemporanei, come aveva già notato Crescimbeni nei suoi Comentarj.5

Un tema coltivato in Italia, da Dante e Petrarca fino a Leopardi, ${ }^{6}$ era quello dell'Italia personificata, regina ridotta a serva, povera e ferita, a causa delle invasioni straniere. Nelle ottave Le lagrime dell'Alpe, della raccolta La selva di cipressi (1640), Margherita, oltre all'Italia personificata, personifica anche i fiumi Po e Dora, nonché l'Alpe, che ha un serto di querce annose e chiome nevose, la quale si lamenta:

L'erba non più le piagge mie riveste, né de' smeraldi suoi colora il colle, poich' ivi d'onde sanguinose e meste, il tutto intorno è ricoperto e molle. Cadono al pian recise le foreste, né più il pino o la quercia i crini estolle, ché, per comporne macchine guerriere, campi aperti si fan le selve intiere.

(VVC, p. 177)

Attraverso il lamento di queste quattro personificazioni che descrivono la distruzione e la morte nei loro territori più colpiti, Margherita crea, in modo originale, scene lugubri ma potentissime di stragi e rovine, deplorando così la devastazione causata dalle guerre fra la Spagna e la Francia, combattute sul suolo italiano.

Altri temi comuni al suo secolo sono i lamenti di amanti infelici: per la partenza della persona amata, per un amore non corrisposto, o per la libertà riacquistata - temi popolarissimi nel mondo musicale a cui apparteneva anche Margherita, virtuosa di canto. Alcuni fra i sonetti di questo tipo sono veri gioielli. Per esempio, quello in cui una donna si rivolge al suo cuore per lamentarsi di non essere riamata, anzi, di essere presa in giro, per la sua passione, dal suo idolo:

Mio cor, che fai, che speri, a che sospiri per chi preda non è dei tuoi pensieri? 
Forse trar dalle stelle i lumi speri, vago del mio penar, dei miei martiri?

Non sperar pace, benché i lumi giri ridendo agli ardor tuoi con modi alteri, scherzan, folle che sei, quegli occhi arcieri per far preda di morte i tuoi desiri.

Arride al tuo dolor il dolce riso, rallegra l'alma sua nel tuo tormento e, per farti penar, ti volge il viso.

Superbo del tuo duol, vive contento e, con folle scherzar di van sorriso, cede le tue speranze all'aria, al vento. (VVC, pp. 67 e 69)

Oppure il sonetto rivolto all'amante infedele, in cui la donna dichiara di essersi liberata dalle catene che la legavano a lui, tema che arriverà al suo apice con $L a$ libertà (rivolta a Nice) di Metastasio:

Son pur finiti, ingrato, i miei tormenti, non sento pene più, né più martiri. Né mai fia che le orecchie o gli occhi giri agli tuoi falsi e perfidi andamenti.

Ritorno alle mie gioie, ai miei contenti, fuggon da me gli pianti e gli sospiri. L'alma ritorna ai soliti desiri. né più si lagna o si querela ai venti.

Né più dei vezzi tuoi, né di tua fede mi curerò giammai, benché fedele, né bramo più da te nulla mercede.

Già ti conosco un falso, un infedele, che natura ti fe' per farti erede d'un animo villan, d'un cor crudele. (VVC, p. 69)

A questi sonetti ci sarebbero da aggiungere quelli per la partenza dell'amante, fra cui spicca Bella donna nella partenza dell'amante, per la sensualità esposta apertamente:

Tu parti, anima mia, ed è pur vero che qui mi lasci abbandonata e sola. 
Chi fia che più m'aita o mi consola, se tu lungi da me cangi pensiero?

Fra le speranze mie sperar dispero,

l'alma col tuo partir da me se n'vola, non oso senza te formar parola e sol dal mio dolor la morte spero.

M'aggiro entro il tuo nido e queste mura piangendo miro, e l'innocente letto dolente premo, fra dolente arsura.

Deh, fa, bell'idol mio, che sia ricetto ad ambe due, con più pietosa cura torna l'anima tua dentro il mio petto. (VVC, pp. 79 e 81 )

Similmente la passione di una donna viene espressa con tinte forti, che ricordano il «viver ardendo e non sentire il male» di Gaspara Stampa (Rime, CCVIII, v. 6.), nel sonetto in cui Margherita crea un bel contrasto fra la giornata buia, nebbiosa, priva di luce, e lo sguardo ardente e luminoso dell'amante, che le brucia l'anima:

Era torbido il cielo e senza venti l'aere nebbioso, ed era il sol oscuro, quando il mio vago sol, lucido e puro, avventò verso me raggi cocenti.

E furono i lumi suoi due fiamme ardenti che m'arser l'alma, ond'io non m'assicuro di viver più, né viver più mi curo se conforto non hanno i miei tormenti.

Amor, o tu m'aita o il crudo affetto dal cor mi svelle e spegni l'empia face. ché goder non si può senza diletto.

Per sì vaga beltà morir mi piace, ma per tomba vorrei quel vago petto ove l'anima mia sepolta giace.

(VVC, p. 71)

Anni più tardi, nella raccolta La selva di Diana (1647), Margherita torna al tema della giornata buia, questa volta di pioggia, creando un sonetto mirabile in cui la pioggia è vista come il pianto della natura, del sole e delle stelle per diventare tutt'uno con il pianto della donna: 
Il cielo è fatto oscuro e senza luce.

il dì tra nembi di terror si mostra.

Febo d'impure macchie il volto inostra

e squallida l'Aurora il corso adduce.

Vacillante la terra a noi produce

pallidi fiori ed alla luce nostra

fa d'atra notte formidabil mostra,

ché il dio dello splendore altrove luce.

E l'aria col suo pianto il pianto mio

grave accompagna ed alle mie procelle

fulmini scote il fulminante dio.

Piange il ciel, piange il sole, piangon le selle

ai miei martiri e, per mio duol più rio

tra i pianti, il dio d'amore vibra facelle.

(VVC, p. 239)

I versi di Margherita non sono affatto brutti, rozzi o mal scritti. Sono invece ben formulati e originali nel descrivere la passione femminile in modo più ardito e realistico a paragone dei suoi contemporanei.

Per concludere: ho scoperto in Margherita una scrittrice versatile, l'unica poetessa italiana veramente barocca, che osò competere con i marinisti e che coltivò anche l'umorismo, l'ironia e la satira con notevole abilità, perfino in situazioni assurde. Pubblicò sei volumi di versi, due libri di prose, tre opere teatrali, due poemi e una specie di Balletto a Cavallo con testo in versi. Fu una donna coraggiosa che dovette combattere contro i tanti pregiudizi della società, spesso ipocrita, della controriforma. In più, come virtuosa del canto, era legata alla vita teatrale, allora considerata immorale. Non tutte le sue opere avranno lo stesso valore letterario, ma anche quelle minori hanno certamente un valore storico come descrizioni di costumi secenteschi, di eventi contemporanei, o di divertimenti barocchi elaborati in modo originale. Mi auguro che le ricercatrici più giovani di me continueranno ad esaminare i vari volumi di Margherita Costa, di cui ho potuto dare solo un assaggino in Voice of a Virtuosa and Courtesan. Di certo, questo libro è servito a risvegliare l'interesse per Margherita, poetessa e scrittrice (di cui si conosceva bene solo la commedia $\mathrm{Li}$ buffoni), come risulta dai lavori apparsi dopo il 2015 e soprattutto dai saggi presentati in questo Convegno Internazionale. 


\section{Note}

${ }^{1}$ Nel 2018 è uscita la traduzione inglese, sotto il titolo The Buffoons, A Ridiculous Comedy, a c. di Sara Diaz e Jessica Goethals.

${ }_{2} \mathrm{Nel} 2018$ è uscita la traduzione inglese, sotto il titolo The Buffoons, A Ridiculous Comedy, a c. di Sara Diaz e Jessica Goethals.

${ }^{3}$ Si noti l'uso dell'aggettivo frale: «sesso frale».

4 Zio di Ferdinando II.

${ }^{5} \mathrm{Si}$ veda VVC, p. 40.

${ }^{6} \mathrm{Si}$ veda Costa-Zalessow 1968.

\section{Bibliografia}

\section{ADIMARI 2009:}

Alessandro ADIMARI, La Tersicore, Firenze, Massi \& Landi, 1637, Ed. moderna a c. di Paola Marongiu, Milano, Res, 2009.

CAPUCCI 1984:

Martino CAPUCCI, Costa, Margherita, in Dizionario biografico degli italiani, Roma, Enciclopedia Treccani, 1984, vol. 30, pp. 232-34.

COSTA-ZALESSOW 1968:

Natalia Costa-ZALESSOW, Italy as a Victim: A Historical Appraisal of a Literary Theme, in «Italica», 45 (1968), 2, pp. 216-40.

COSTA-ZALESSOW 1982:

Natalia CosTA-ZALESSOW, Scrittrici italiane dal XIII al XX secolo: testi e critica, Ravenna, Longo, 1982.

COSTA-ZALESSOW 2008:

Natalia CosTA-ZALESSOW, Margherita Costa, in Albert N. MANCINI, Glenn Palen PIERCE, Dictionary of Literary Biography. Seventeenth-century Italian poets and dramatists, Bruccoli, Clark Layman, 2008, vol. 339, pp. 113-18.

COSTA-ZALESSOW 2015:

Natalia COSTA-ZALESSOW, Voice of a Virtuosa and Courtesan. Selected poems of Margherita Costa, New York, Bordighera Press, 2015.

COSTA-ZALESSOW 2019:

Natalia CosTA-ZALESSOw, Cortegiano ravveduto: satira di Margherita Costa, in «Letteratura italiana antica: rivista annuale di testi e studi», XX (2019), pp. 579-91.

CROCE 2003:

Benedetto CROCE, Donne letterate nel Seicento, in Nuovi saggi sulla letteratura italiana del Seicento, Napoli, Bibliopolis, 2003, pp. 165-82.

DIAZ-GOETHALS 2018:

Margherita COSTA, The buffoons: a ridiculous comedy. A bilingual edition, a c. di Sara E. Díaz, Jessica Goethals, Toronto, Arizona Center for Medieval and Renaissance Studies, 2018. 
MARINO 2007:

Giovan Battista MARINO, La Lira, a c. di Maurizio Slawinski, Torino, Edizioni RES, 2007, vol. 2, pp. 98-102.

MEGALE 1988:

Teresa MEgale, La commedia decifrata. Metamorfosi e rispecchiamenti in Li Buffoni di Margherita Costa, in «Il castello di Elsinore», 2 (1988), pp. 64-76.

STAMPA 1954:

Gaspara STAMPA, Rime, Milano, BUR, 1954. 